\title{
Experiencia en cursos de capacitación en ecotomografía abdominal en patologías de urgencia*
}

\author{
Drs. DAVID LAGOS D. ${ }^{1}$, XIMENA FREITTE B. ${ }^{2}$, GRACIELA RIVERO M. ${ }^{2}$, \\ FERNANDO BOZINOVIC A. ${ }^{2}$, MANUEL ÓRDENES V. ${ }^{3}$, JORGE RODRÍGUEZ G. ${ }^{4}$
}

1 Servicio de Cirugía Hospital de Quillota.

Servicio de Cirugía Hospital de Quilpué.

Servicio de Cirugía Hospital Dr. Gustavo Fricke, Viña del Mar.

Servicio de Cirugía Hospital de San Fernando.

Chile.

\section{Ultrasound of the acute abdomen performed by surgeons}

\section{Introducción}

La realización de ecotomografías, por cirujanos, ha sido motivo de numerosas publicaciones, por 20 o más años, en Europa y en EE.UU., especialmente después de la comunicación de Rozycky, en la que se entregan conceptos básicos de la llamada ecotomografía FAST ${ }^{1-4}$.

El adiestramiento de los cirujanos y residentes en el uso diagnóstico de ultrasonido, se ha incorporado como un nuevo objetivo en centros formadores y sociedades científicas, con énfasis en adquirir habilidades y destrezas para la atención de pacientes en la Urgencia y Trauma. Desde 1998 se exige en Alemania, como parte del curriculum de la formación quirúrgica, el aprendizaje de ecografía además de formar parte de la docencia de pregrado ${ }^{2}$. Por su parte, el Colegio Americano de Cirujanos, ha elaborado material educativo en este sentido, el que se ofrece a sus integrantes y a residentes de la especialidad. Además ha regulado la formación y la práctica por parte de los cirujanos ${ }^{5}$. Su uso en el intra-operatorio, tanto usado por vía laparoscópica como en la cirugía abierta, ha tenido un desarrollo paralelo.

Ha habido una creciente utilización de la ecografía en situaciones de emergencia, en rescate de urgencias, en medicina de guerra, etc, lo que se ha consolidado con la aparición de equipamiento más versátil, de menor costo, de fácil operabilidad y excelente resolución.

En Latinoamérica sin embargo, y en particular en Chile, las instituciones formadoras, Escuelas de Medicina o las Sociedades Científicas, no incluyen a la ecografía como parte de la formación de pre o post grado de cirujanos o internistas.

Los becados de cirugía y los cirujanos ya formados, no tienen facilidades para aprender y adiestrarse en forma sistemática en el uso de la ecografía. Salvo excepciones, la docencia en este tema es más bien esporádica, no sistematizada y se entrega a la iniciativa de algunos cirujanos interesados en compartir su experiencia, capacitando a otros. Por el contrario, habitualmente se la ha considerado en el ámbito de los servicios de radiología e imágenes, y su realización a cargo de radiólogos. Sin embargo, su utilización en ginecoobstetricia, en vascular periférico, en cardiología, como un valioso agregado de la endoscopía, en la práctica de anestesia, nos demuestra que se trata de una técnica de uso mucho más amplio y que incluso podría considerarse hoy en día como una extensión del examen físico.

Motivados por la convicción que el cirujano de urgencias, debe tener la mejor capacitación posible y poner al alcance de sus pacientes el oportuno be-

*Recibido el 17 de Noviembre de 2009 y aceptado para publicación el 22 de Diciembre de 2009.

Correspondencia: Dr. David Lagos D.

Concepción 1050, Quillota, Chile.

E-mail: davidr.lagos@gmail.com 
neficio de los avances tecnológicos, permitiendo un mejor enfoque terapéutico, un grupo de "CirujanosEcografistas" de la V ${ }^{a}$ Región organizó un curso para adiestrar a cirujanos de urgencias y a residentes de cirugía en ecotomografía, con el objetivo de mejorar sus capacidades resolutivas. Los conocimientos y experiencia de los integrantes del grupo, permitieron tener la certeza de entregar los conceptos necesarios y suficientes para mejorar la atención del paciente de urgencias.

En la presente comunicación se da cuenta de la evaluación después de 5 años de trabajo y 6 cursos de capacitación en ecotomografía para cirujanos.

El objetivo ha sido evaluar la factibilidad de realizar docencia hecha por cirujanos a cirujanos generales en ecotomografía de urgencias y los resultados a largo plazo de la utilización de las destrezas adquiridas.

\section{Material y Método}

Se programó un Curso Modular de Ecotomografía, considerando los fundamentos de la física del ultrasonido y técnicas básicas del examen ultrasónico abdominal en temas de patología quirúrgica de Urgencias, con el apoyo de la Filial V ${ }^{\mathrm{a}}$ Región de la Sociedad de Cirujanos de Chile, el Servicio de Salud de Viña del Mar/Quillota y de los Hospitales de Quilpué y Quillota, donde se realizaron los Cursos teórico/prácticos.

Los docentes fueron seleccionados entre cirujanos con adiestramiento en ecotomografía abdominal obtenido en Chile o en el extranjero y que realizan en forma habitual este tipo de exploraciones en sus establecimientos (Hospitales de San Fernando, Quilpué y Quillota) tanto en los Servicios de Cirugía como en Unidades de Emergencia.

Estuvo dirigido a Cirujanos de Unidades de Emergencia de la $\mathrm{V}^{\mathrm{a}}$ Región y a becados de la especialidad.

Se obtuvo el apoyo de empresas privadas en insumos y equipos de ultrasonido. Los pacientes reclutados para la realización de ecotomografías, fueron seleccionados de las listas de espera de los hospitales donde se realizó el Curso.

Los pacientes dieron su consentimiento para la realización de los exámenes, al informárseles de los objetivos y la supervisión por expertos de los procedimientos a realizar. Se incluyó además pacientes hospitalizados, de la Unidad de Emergencia y a voluntarios sanos.

En cada curso, se realizó una prueba diagnóstica inicial, para evaluar el grado de conocimiento de las imágenes ecográficas, la cual se repitió al finalizar, con las mismas imágenes, para que los alumnos midiesen su grado de aprendizaje.

La capacitación consistió en 20 horas lectivas de actividades académicas, 5 de tipo teórico y 15 de tipo práctico. Cada "Cirujano-Monitor", tuvo a su cargo 3 ó 4 alumnos y un ecógrafo, cada grupo examinó un mínimo de 20 pacientes en base a enseñanza directa monitor/educando.

Con el objeto de evaluar el grado de utilización de conocimientos y destrezas adquiridas, se confeccionó una encuesta a los alumnos, midiendo el impacto que la capacitación en ecotomografía había producido en su práctica quirúrgica cotidiana, como también la opinión sobre el adiestramiento en ecotomografía de urgencias y la forma de adquirirlo (Anexo 1).

Se incluyeron en el estudio a los participantes de los primeros 6 cursos, ya que tenían un tiempo de seguimiento de más de 6 meses. No se incluyó a participantes desde agosto de 2009 en adelante por dicha razón.

\section{Resultados}

Entre julio de 2004 y diciembre de 2008 se realizaron 6 cursos modulares, con una participación de 86 alumnos en total, cuya condición académica se señala en la Figura 1.

Se recibió un total de 42 respuestas a la encuesta diseñada. (48,8\% del total), las que constituyen la base de esta comunicación. Figura 2 .

La totalidad de los encuestados, considera que el curso aportó conocimientos útiles y prácticos para el trabajo quirúrgico. Salvo 2 encuestados, todos consideraron que debieran ser re-actualizados periódicamente. Figura 3.

E1 76\% de quienes respondieron, afirma realizar ecografías en forma regular. Figura 4.

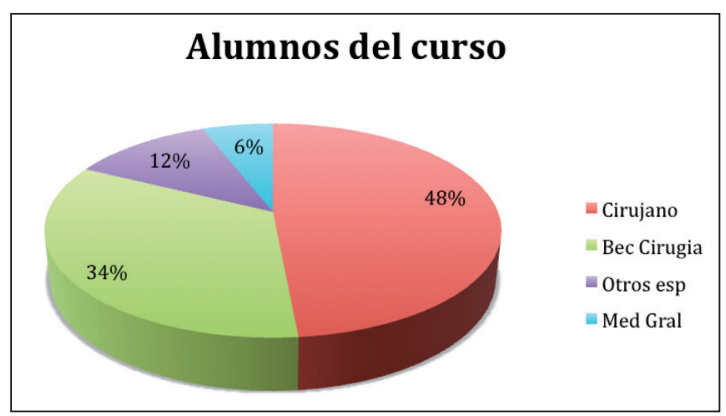

Figura 1. Condición académica y profesional de alumnos participantes en los cursos de ecotomografías $(n=86)$. 


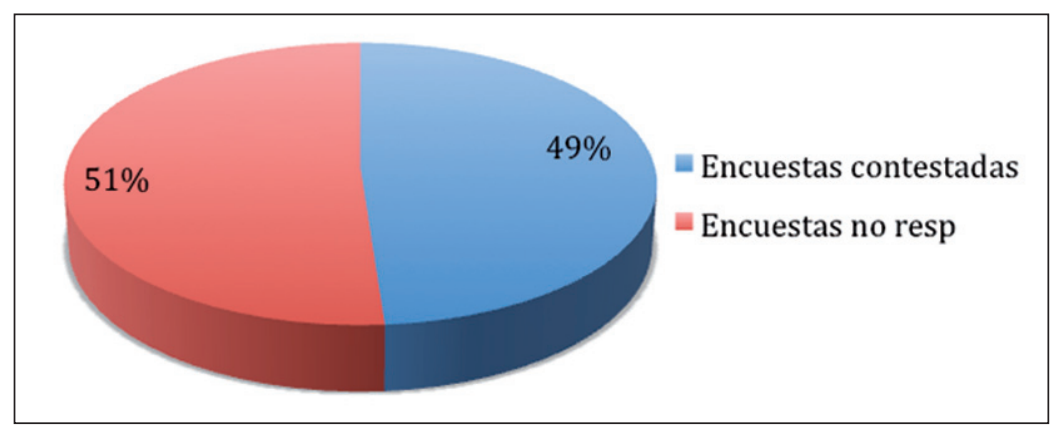

Figura 2. Relación entre encuestas enviadas y respondidas por parte de los alumnos participantes en cursos de ecotomografía.

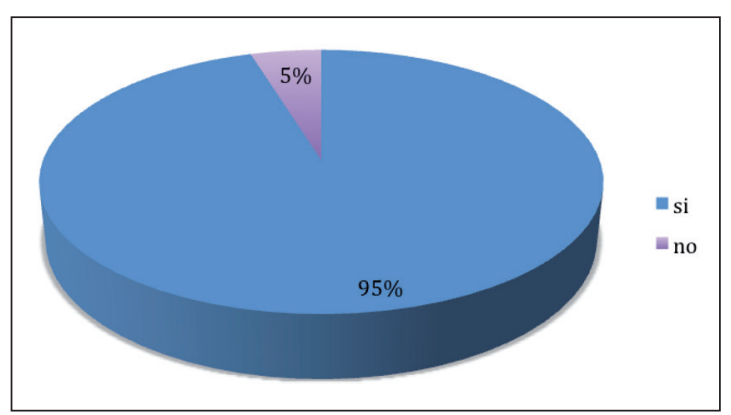

Figura 3. Necesidad manifestada de reciclaje periódico de conocimientos $(n=42)$.

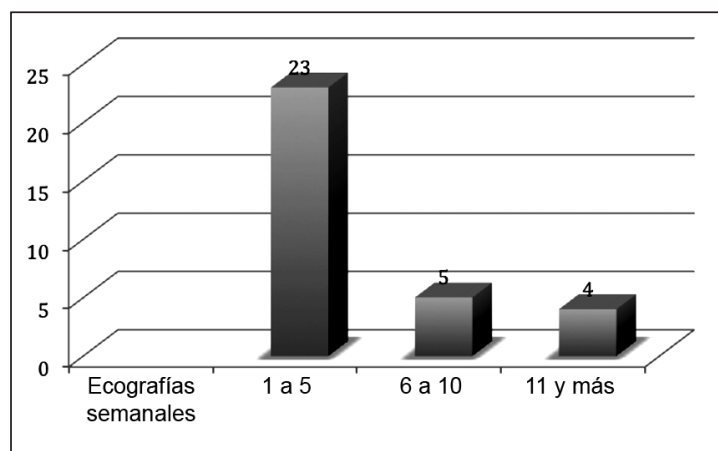

Figura 5. Número de exámenes semanales realizados por alumno posterior a capacitación.

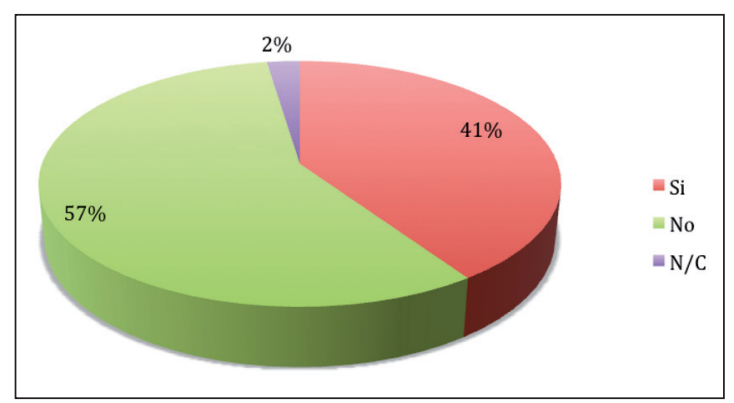

Figura 6. Necesidad manifestada de otros conocimientos no entregados en el curso $(n=42)$.

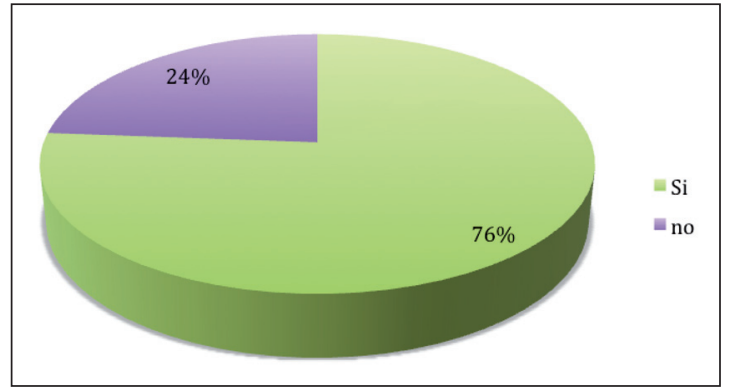

Figura 4. Realización de ecografías en forma regular posterior a curso $(n=42)$.

Entre quienes realizan estas exploraciones, el $74 \%$ efectúa entre 1 a 5 por semana, el $16 \%$ entre seis y diez, y el 10\% 11 o más por semana. Figura 5.

Diecisiete alumnos ( $41 \%$ de los que respondieron la encuesta), mencionan la necesidad de incorporar nuevos conocimientos, como doppler vascular, procedimientos guiados por ecografía, examen de partes blandas, trauma, testículo agudo, etc. Figura 6.

El 80\% menciona la necesidad de formación en ecografía, durante la formación como parte del currículum del cirujano general. El 20\% restante señala además que debería contemplarse docencia sobre este tema en el pre-grado. Figura 7.

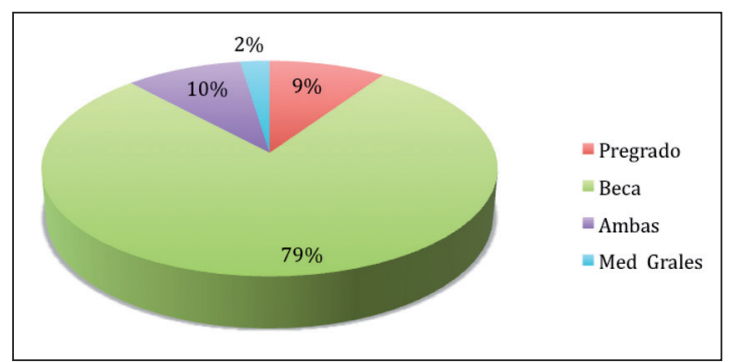

Figura 7. Entrega de conocimientos en ecografía $(\mathrm{n}=42)$. 


\section{Discusión}

En la actualidad la ecotomografía ha llegado a ser parte del examen físico del abdomen, en especial en el servicio de Urgencias donde la toma de decisiones se puede ver favorecida con la información adicional que se obtiene con su uso.

Hasta ahora, la formación "clásica" de los cirujanos ha puesto mayor énfasis en la realización de procedimientos terapéuticos y no tanto en aquellos diagnósticos. Por esta razón, no nos ha preocupado el desarrollar destrezas en áreas que inconscientemente consideramos del ámbito de otra especialidad.

En los Cursos ATLS, se señala a la "EcoFast" como fundamental en la evaluación del paciente traumatizado, pero no se ha establecido el proceso formativo para llenar ese vacío de conocimientos.

Es llamativa la falta de capacitación en este tema en los programas de pre y post-grado de cirugía de nuestro país. Salvo contadas excepciones, no conocemos planes y programas dentro de la malla curricular, para que becados de cirugía se entrenen en manejar ecógrafos.

Especialistas como los gineco-obstetras han incluido desde hace tiempo, el adiestramiento en ecotomografía como parte de sus programas de formación y el ultrasonido ha reemplazado al estetoscopio de Pinard. Los cirujanos vasculares, cardiólogos, oftalmólogos, anestesistas, gastroenterólogos (Eco-endoscopía), nos han demostrado las ventajas de disponer de esta herramienta como ayuda cotidiana en la mejoría de calidad de nuestro trabajo.

Rozycki $^{4}$, señala que a su juicio, los "dominios exclusivos" han desaparecido y que no hay especialidades que deban ejercer derechos sobre determinadas tecnologías. Afirma que las consideraciones clínicas deben estar por encima de consideraciones económicas o políticas y, al menos en EE.UU., los cirujanos publican de manera destacada su experiencia en capacitación para y por cirujanos ${ }^{6}$. En España se publicó un texto editado por un profesor de Medicina Interna ${ }^{7}$.

Los cirujanos de los Servicios de Urgencias entienden aún mejor las ventajas de un adiestramiento que permita tomar las mejores decisiones documentadas en pacientes complejos, como ya se ha demostrado en otras latitudes ${ }^{8-14}$.

Conociendo esta realidad y la existencia de cirujanos con conocimientos y destrezas capaces de romper paradigmas, podemos afirmar que nuestra experiencia regional nos demuestra que:

a. Existe alto interés y necesidad de formación en ecotomografía abdominal en los cirujanos generales y becados de cirugía. b. Los cirujanos están dispuestos a adquirir más capacidades para enfrentar el trabajo diario, especialmente en Urgencias.

c. Un porcentaje considerable de quienes han aprendido la técnica, la usan regularmente en su trabajo cotidiano.

d. Los cirujanos con estas destrezas adquiridas, son capaces de adiestrar a su vez a otros cirujanos y becados de cirugía.

La industria pone a nuestro alcance equipos de ecografía cada vez más versátiles, compactos y de menor costo. La realización de ecotomografías no tiene contraindicaciones y se puede repetir tantas veces como se estime conveniente.

Los cirujanos conocen detalladamente la anatomía normal y patológica del abdomen y les resulta sencillo reconstruir imágenes de vísceras a partir de imágenes ultrasónicas bidimensionales.

En especial los cirujanos de urgencias, requieren de respuestas estructuradas a la necesidad de aprendizaje de la ecotomografía en este ámbito y las buscan dirigidamente. Torsión testicular, hernia atascada, obstrucción intestinal, cálculo coledociano y dilatación de la vía biliar, tubario complicado, apendicitis y colecistitis aguda, líquido libre abdominal etc, son condiciones en las que el ultrasonido presta una ayuda invaluable por lo que no se entiende la demora que mantenemos en el adiestramiento de nuestros colegas de urgencia.

Los alumnos de nuestros cursos han señalado la utilidad del nuevo conocimiento adquirido -independiente si realizan estudios ecográficos o no- y la necesidad de incorporarlo en la formación como especialistas y que al igual que otros conocimientos se esté permanentemente actualizando.

Esta puede ser una tarea de la Sociedad de Cirujanos o de los centros de formación de post-grado.

\section{Agradecimientos}

Nuestra gratitud a quienes han contribuido de manera significativa al éxito de cada uno de los cursos realizados:

- Todos los pacientes de los Hospitales de Quilpué y Quillota que generosamente aceptaron someterse a exámenes para que los especialistas de hoy y mañana puedan entregarles una mejor atención.

- Electromed e International Clinics que facilitaron los equipos gentilmente, asumiendo los riesgos de traslado y manipulación por alumnos.

- Laboratorios Saval y Andrómaco por su apoyo con insumos y material docente.

- El personal de apoyo de ambos hospitales sin cuya labor no habría sido posible contar con pacientes, su traslado, realización de informes, etc. 
- La secretaria de la Sociedad de Cirujanos de la V región.

- La Sociedad de Cirujanos de Chile por su apoyo y confianza en la iniciativa.

- A todos los alumnos que han confiado en el equipo docente.

\section{Referencias}

1. Holm H. Interventional Ultrasound. Editorial Munksgaard, Copenhagen, 1985.

2. Frankel H. Ultrasound for Surgeons. Vademecum Landes BioScience 2005.

3. Hofer M. Ultrasound Teaching Manual 1999.

4. Rozycki GS. Surgeon-performed ultrasound: its use in clinical practice. Ann Surg 1998; 228: 16-28.

5. Am College of Surgeons, Division of Education.

6. An update in surgeon-performed ultrasound. Surg Clin N A 2004; 84: 953-1179.

7. Roca Martínez FJ. Ecografía Clínica del Abdomen. Primera edición 1879, editorial JIMS S.A.

8. Spence SC, Teichgraeber D, Chandrasekhar C. Emer- gent right upper quadrant sonography. J Ultrasound Med 2009; 28: 476-496.

9. Krettek C. Ultrasound for abdominal and thorax trauma. Unfallchirurg 2008; 111: 957.

10. Wening JV, Tesch C, Huhnholz J, Friemert B. The value of sonography in traumatology and orthopedics: Part 2: Emergency diagnostics in blunt abdominal and thoracic trauma. Unfallchirurg 2008; 111: 958-964.

11. Brooks A, Davies B, Smethhurst M, Connolly J. Prospective evaluation of non-radiologist performed emergency abdominal ultrasound for haemoperitoneum. Emerg Med J 2004; 21: 580-581.

12. Catan F, Altamirano C, Salas C, Novoa R, Castro JP, Lagos C, y cols. Ecografía realizada por cirujanos en el manejo de pacientes con trauma. Rev Med Chile 2002; 130: 892-896.

13. Lindelius A,Torngren S, Sonden S, Pettersson H, Adami J. Impact of surgeons-performed ultrasound on diagnosis of abdominal pain. Emerg Med J 2008; 25: 486-491.

14. Eiberg JP, Grantcharov TP, Eriksen JR, Boel T, Buhl $\mathrm{C}$, Jensen D, et al. Ultrasound of the acute abdomen performed by surgeons in training. Minerva Chir 2008; 63: 17-22.

\section{Anexo 1. Encuesta a cirujanos participantes en el curso de ecotomografía en urgencias}

1.- Cuál era su situación académica al momento de realizar el curso.

a.- Alumno de pregrado

b.- Becado de Cirugía

c.- Cirujano (tiempo de práctica de la especialidad)

d.- Otro (detallar)

2.- Después de hecho el curso, realiza ecotomografías en su práctica quirurgica electiva o de urgencia.

a.- Sí

b.- No

3.- En caso afirmativo ¿cuántas ecotomografías realiza a la semana?

a.- 1 a 5

b.- 6 a 10

c.- más de 10

4.- Considera que el curso de ecotomografía le entregó conocimientos útiles y prácticos para su trabajo médico.

a.- Sí

b.- No

5.- Cree Ud. que debiera reciclar sus conocimientos y destrezas ecográficos periódicamente

a.- Sí (cada cuánto tiempo)

b.- No

6.- Considera que necesita algún conocimiento específico no entregado en el curso

a.- Sí (cuál)

b.- No

7.- Cree Ud. que la ecotomografía debiera ser materia de cursos formales en:

a.- Pregrado

b.- En la beca de cirugía

Agradeceríamos cualquier sugerencia o comentario que considere oportuno para ser considerado en cursos futuros. 